\title{
DEPENDENCIA QUÍMICA POR EL CONSUMO DE MARIHUANA: DEL PLACER A LA ENFERMEDAD. UN GRAVE PROBLEMA EN SALUD PÚBLICA EN BRASIL
}

\section{ARTÍCULO ORIGINAL}

DIAS, Amanda de Araújo1', DIAS, Édina Lúcia de Araújo², OLIVEIRA, Ciane Martins de $^{3}$, DENDASCK, Carla Viana ${ }^{4}$, OLIVEIRA, Euzébio de ${ }^{5}$

DIAS, Amanda de Araújo Dias. Et al. Dependencia química por el consumo de marihuana: del placer a la enfermedad. Un grave problema en salud pública en Brasil. Revista Científica Multidisciplinar Núcleo do Conhecimento. Año 06, Ed. 03, Vol. 11, pp. 78-86. Marzo de 2021. ISSN: 2448-0959, Enlace de acceso: https://www.nucleodoconhecimento.com.br/salud/consumo-de-marihuana, DOI: 10.32749/nucleodoconhecimento.com.br/salud/consumo-de-marihuana

\section{RESUMEN}

Introducción: El consumo de marihuana ha aumentado a nivel mundial, siendo la droga ilícita más utilizada en el mundo. Se estima que entre 167 y 315 millones de personas de entre 15 y 64 años utilizaron alguna droga ilícita en la última década. En Brasil, aproximadamente 7,5 millones de estudiantes universitarios, distribuidos en aproximadamente 2.400 instituciones hacen uso de la hierba. Método: Se realizó un estudio descriptivo basado en una revisión de la literatura. La investigación se llevó a cabo a través de la base de datos Scielo y la biblioteca de salud virtual (BVS).

\footnotetext{
${ }^{1}$ Académico Médico en el Centro Universitario del Estado de Pará - CESUPA.

2 Psicólogo. Postgrado en Salud Mental en escola Superior da Amazônia - ESAMAZ.

${ }^{3}$ Doctor en Genética Médica. Profesor e investigador del Centro Universitario del Estado de Pará (CESUPA).

${ }^{4}$ Teólogo, Doctor en Psicoanálisis Clínica. Ha estado trabajando durante 15 años con Metodología Científica (Método de Investigación) en la Guía de Producción Científica de estudiantes de Maestría y Doctorado. Especialista en Investigación de Mercado e Investigación centrado en la salud.

${ }^{5}$ Doctorado en Medicina/Enfermedades Tropicales. Profesor e investigador de la Universidad Federal de Pará UFPA.
}

RC: 80474

Disponível em: https://www.nucleodoconhecimento.com.br/salud/consumo-de- 
Resultados $\mathrm{Y}$ discusión: Con respecto a los efectos de la marihuana, en algunos estudios se reportan síntomas relacionados con el uso recreativo y el abuso de esta droga. Sin embargo, a nivel académico, los efectos del consumo de marihuana se pueden dividir en síntomas agudos y crónicos. Efectos agudos se clasifican como euforia, efectos físicos y efectos psíquicos como depresión, alucinación, ilusión, somnolencia y deterioros de la concentración y memoria a corto plazo. Conclusión: Se ha demostrado alguna evidencia que puede explicar por qué los individuos eligen usar marihuana. Más a menudo se desean los efectos agudos de la euforia, el placer y la relajación, en contraste, se observa que los daños asociados con este uso terminan superando los beneficios ilusorios que ofrece la droga, lo que conduce al placer a convertirse en una enfermedad.

Palabras clave: Marihuana, consumo, placer, enfermedad.

\section{INTRODUCCIÓN}

El hábito de consumir drogas ha estado presente y establecido en las sociedades desde la antigüedad, y el primer registro de consumo de marihuana (cannabis) se remonta al menos a diez mil años, en los que hay registros de cultivo y uso por diversas civilizaciones de todo el mundo, siendo la más antigua de Asia y Oriente Medio, llegando más tarde a África, América y otras regiones (ROSA; ROSA, 2018).

Se estima que entre 167 y 315 millones de personas de entre 15 y 64 años utilizaron alguna droga ilícita en la última década, lo que representa entre el 3,6 y el 6,9\% de la población mundial. En este contexto, el consumo de marihuana ha aumentado a nivel mundial, siendo la droga ilícita más utilizada en el mundo. Se estima que la marihuana se consume entre 125 y 203 millones de personas en la actualidad, con la mayor prevalencia en África Central y Occidental (GARCIA, 2014).

En Brasil, aproximadamente 7,5 millones de estudiantes universitarios, distribuidos en aproximadamente 2.400 instituciones utilizan la hierba, y el uso de la sustancia es

RC: 80474

Disponível em: https://www.nucleodoconhecimento.com.br/salud/consumo-de- 
una práctica frecuente, muy discutida por los medios laicos y analizada por algunos estudios científicos (GARCIA, 2014; FERNANDES et al., 2017).

Dado que el uso de la marihuana es cada vez más frecuente en nuestro entorno social, es extremadamente importante que se realicen estudios con el fin de ampliar el conocimiento sobre sus efectos químicos, psicológicos y sociales debido a la importancia que presentan.

Además, en los últimos años se ha debatido sobre los pros y los contras relacionados con la legalización de esta droga, y para que tanto los profesionales como la población puedan dar su opinión, primero es necesario entender cómo la marihuana modifica el cuerpo a corto y largo plazo (COUTINHO; ARAÚJO; GONTIÈS, 2004).

En este contexto, este trabajo se justifica por ser creado con el fin de refinar los conocimientos existentes sobre el uso de la marihuana y actualizar a la sociedad académica sobre el proceso de placer y enfermedad al que esta droga está directamente vinculada.

Esta revisión tiene como objetivo abordar los principales aspectos de la dependencia química por el consumo de marihuana, así como conceptos sobre las sensaciones placenteras de usar esta droga y cómo está contribuyendo al deseo de continuar su uso. También es de interés para este artículo discutir las consecuencias físicas y psicológicas que la marihuana origina en el cuerpo caracterizando un proceso de enfermedad por su uso prolongado, contribuyendo a una mejor información sobre las razones para no usar esta droga.

\section{MATERIAL Y MÉTODO}

La metodología utilizada en el presente estudio fue un estudio descriptivo basado en una revisión de la literatura. La investigación bibliográfica se llevó a cabo a través de

$\mathrm{RC}: 80474$

Disponível em: https://www.nucleodoconhecimento.com.br/salud/consumo-de- 
la base de datos Scielo y la Biblioteca Virtual de Salud (BVS). Para registrar los resúmenes portugueses en tales bases, se utilizaron los siguientes términos: marihuana, placer y enfermedad. Después de este proceso inicial, los títulos fueron leídos, y luego los resúmenes, para seleccionar las publicaciones que interesaban a la investigación, en base a los requisitos establecidos como criterios de inclusión y exclusión. Esta investigación tuvo como criterios de inclusión artículos publicados en el período de 2002 a 2020, en lengua portuguesa, que abordan los temas relacionados con la marihuana y sus principales efectos fisiológicos y patológicos en el cuerpo humano, con respecto al cuerpo y la mente. Los criterios de exclusión eran artículos publicados en una lengua extranjera que no cumplían los criterios de inclusión. Finalmente, los artículos seleccionados fueron analizados y originaron la escritura de la presente obra.

\section{REVISIÓN Y DISCUSIÓN DE LA LITERATURA}

\subsection{CONCEPTOS BÁSICOS RELACIONADOS CON LA MARIHUANA}

La marihuana es una hierba cuyo nombre científico es Cannabis. En el cannabis latino significa cáñamo, que llama la familia de plantas, y hay tres tipos de especies de esto, siendo sativa, indica y ruderalis (FERRARI, 2016). Las especies, sativa e indica son las más utilizadas, tanto para fumar, como para diversas aplicaciones en cocina y medicina (CORTEZ, 2009).

Durante siglos la población ha estado utilizando hierba de marihuana con fines medicinales, por ejemplo, los chinos señalaron las propiedades medicinales de la marihuana como anticonvulsivo, calmante y analgésico. El potencial de sus propiedades medicinales en el tratamiento contra algunas enfermedades ya está científicamente demostrado, requiriendo más estudios para analizar sus efectos en este ámbito (RIBEIRO; MARQUES, 2008).

RC: 80474

Disponível em: https://www.nucleodoconhecimento.com.br/salud/consumo-de- 
Cannabis sativa contiene alrededor de 400 productos químicos, al menos 60 de ellos alcaloides, conocidos como cannabinoides. El principal componente psicoactivo de la planta es el tetrahidrocannabiol (THC), uno de los compuestos de la hierba, que también consiste en otros cannabinoides como cannabidiol (CBD), cannabinol (CBN) y tetrahidrocanabivarin (THCV).Estos se dividen en psicoactivos (delta-8-THC, delta9-THC y 11-hidroxi-delta-9-THC) y no psicoactivos (cannabidiol y cannabinol). Delta9-THC es conocido por ser el más potente de los cannabinoides (SOLOWIJ; PESA, 2008; CORTEZ, 2009).

\subsection{EL EFECTO Y EL PLACER}

La principal forma de usar hierba de marihuana es a través del tabaquismo y la biodisponibilidad del THC cuando se fuma es de aproximadamente el $20 \%$. Otras formas de uso se pueden realizar a través del Haxixe que también se puede fumar y tiene concentraciones más altas de THC, y aceite de hashoil o cannabis, que es la forma líquida y más potente de la droga intravenosa. Cuando se inhala el humo generado por la hierba quemada llega a los alveolos pulmonares, penetra la circulación sanguínea y llega al cerebro en minutos (CORTEZ, 2009).

Las tasas de absorción oral son más altas (90 a 95\%) (30 a 45 minutos) en relación con la absorción pulmonar (50\%). Los efectos farmacológicos por absorción pulmonar pueden tardar entre 5 y 10 minutos en comenzar (SOLOWIJ; PESA, 2008; CORTEZ, 2009).

Los efectos máximos causados por la droga ocurren en 30 minutos y termina después de aproximadamente dos o tres horas (CORTEZ, 2009).

Los cannabinoides se depositan principalmente en órganos ricos en tejido adiposo, como el cerebro y los testículos, y por lo tanto algunos usuarios pueden presentar signos y síntomas de intoxicación hasta 12-24h debido a la liberación lenta de estas sustancias por adipocitos (SOLOWIJ; PESA, 2008).

RC: 80474

Disponível em: https://www.nucleodoconhecimento.com.br/salud/consumo-de- 
El tiempo de vida media del THC puede variar de 20h a 10 a 13 días y la eliminación total puede tomar hasta 30 días. Por lo tanto, si un individuo utiliza marihuana repetidamente a intervalos más pequeños que este, la concentración de THC en su sangre aumentará, causando efectos más intensos (SOLOWIJ; PESA, 2008; CORTEZ, 2009).

Los cigarrillos de marihuana, ampliamente encontrados, aunque prohibidos en algunos países como Brasil, también se conocen como "a base", que consisten en aproximadamente 0.3-1g de marihuana con una concentración de 1-15\% delta-9THC (2.5 a $150 \mathrm{mg}$ de THC). En este contexto, se observa que la producción de efectos de euforia se produce con una concentración mínima de $1 \%$ THC 01 cigarrillo de 2 a $5 \mathrm{mg}$. Y contrariamente a lo que muchos piensan, los síntomas de intoxicación ya surgen unos minutos después de su uso (RIBEIRO; MARQUES, 2008; SOLOWIJ; PESA, 2008).

En cuanto a los efectos de la marihuana, en algunos estudios se reportan síntomas relacionados con el uso recreativo y abuso de esta droga. Sin embargo, a nivel académico, los efectos del consumo de marihuana se pueden dividir en síntomas agudos y crónicos. Los efectos agudos se clasifican en eufóricos (aumento del deseo sexual, sensación de lentitud en el tiempo, mayor autoconfianza y grandiosidad, risa desmotivada, hilaridad, relajación y mayor percepción de colores, sonidos, texturas y sabor), efectos físicos (taquicardia, conjuntiva hiperemia, sequedad de boca, hipotermia, mareos, retraso psicomotor, descoordinación motora, disminución de la agudeza visual, aumento de la agudeza auditiva, broncodilatación, aumento del apetito, tos y midriasis) y efectos psíquicos (despersonalización, desrealización, depresión, alucinaciones, ilusión, somnolencia, deterioro de la concentración y la memoria a corto plazo, ataques de pánico y paranoia) (SOLOWIJ; PESA, 2008; CORTEZ, 2009).

$\mathrm{RC}: 80474$

Disponível em: https://www.nucleodoconhecimento.com.br/salud/consumo-de- 


\subsection{ABUSO Y ENFERMEDAD}

Algunos autores inducen que el consumo de sustancias psicoactivas ha estado pasando por tiempos y culturas, siendo utilizado en rituales religiosos, como método terapéutico y en la búsqueda del placer, haciendo hincapié en el hedonismo como referencia en este proceso (COUTINHO; ARAÚJO; GONTIÈS, 2004).

En un período a corto plazo, se pueden observar los efectos nocivos del consumo de marihuana, como los déficits motores, al realizar tareas como conducir, por ejemplo; y problemas cognitivos, concentración y memoria y, en consecuencia, dificultades de aprendizaje. Por otro lado, uso a largo plazo puede estar marcado por la tos crónica, inmunidad alterada, niveles reducidos de testosterona y el desarrollo de enfermedades mentales como esquizofrenia, depresión, ataques de pánico, ansiedad, irritabilidad, desmotivación por vida, alucinaciones y despersonalización. Cuanto mayor sea la dosis consumida, más intensos serán los efectos y las tasas de enfermedades. La dependencia del consumo de marihuana se produce en la medida de su consumo. Los efectos adversos incluyen amnesia, falta de atención, problemas motores, generación de crisis de dependencia y retirada (RIBEIRO; MARQUES, 2008; SOLOWIJ; PESA, 2008).

Algunos estudios muestran disfunción cognitiva en los usuarios de cannabis durante la intoxicación, varias horas después del acto de fumarlo, después de unos días y daños duraderos durante más de 1 mes después de la interrupción del uso. La cronicidad de las alteraciones cognitivas depende del organismo, dosis y periodicidad que el individuo hace uso de la hierba, pero es importante destacar que desde el primer uso de la droga y después de la aparición de la acción THC, estas pérdidas psíquicas ya se manifiestan (RIBEIRO; MARQUES, 2008).

Las relaciones entre el rendimiento cognitivo y la frecuencia del consumo de cannabis pueden indicar un efecto residual de intoxicación aguda o crónica que probablemente se disiparía con el cese de uso. Algunos estudios informan que la

$\mathrm{RC}: 80474$

Disponível em: https://www.nucleodoconhecimento.com.br/salud/consumo-de- 
recuperación de la función cognitiva no está completamente dilucidada, pero en algunas fuentes se encuentra que la recuperación puede ocurrir después de 1 mes de abstinencia o no ocurre en 1 mes o la recuperación puede ser sólo parcial (RIBEIRO; MARQUES, 2008; SOLOWIJ; PESA, 2008).

El uso de marihuana también puede resultar en cambios estructurales en el cerebro, como la reducción en los volúmenes del hipocampo y la amígdala, y todavía no se puede afirmar que estos efectos son reversibles. Pero a través de este proceso se puede entender la verdadera razón por la que el deterioro cognitivo se detecta con mayor frecuencia en la memoria, función ejecutiva, atención y control inhibidor (SOLOWIJ; PESA, 2008).

En este contexto, es interesante observar que el sujeto que utiliza marihuana puede o no tener una razón aparente para realizar dicho uso, y entre los principales factores estimulantes para el consumo de la droga se puede mencionar: escapar de los problemas, curiosidad, búsqueda de placeres e influencia social.

\section{CONCLUSIÓN}

Se puede observar que el uso de la marihuana implica una serie de efectos agudos y crónicos, así como residuales, relacionados con el mecanismo de acción del THC. Además, los usuarios de esta droga a menudo tienen problemas que afectan su vida familiar, profesional y social.

En este contexto, es interesante observar que el sujeto que utiliza marihuana puede o no tener una razón aparente para realizar el uso, pero lo que despierta el deseo de promover estudios en el puente entre el placer y la enfermedad es lo que está detrás de la búsqueda de la sustancia, un hecho que está más allá de los ojos del usuario y el consciente.

RC: 80474

Disponível em: https://www.nucleodoconhecimento.com.br/salud/consumo-de- 
En el presente estudio, se demuestra cierta evidencia que puede explicar las razones por las que los individuos eligen usar marihuana. Más a menudo se desean los efectos agudos de la euforia, el placer y la relajación, en contraste, se observa que los daños asociados con este uso terminan superando los beneficios ilusorios que ofrece la droga, lo que conduce al placer a convertirse en una enfermedad.

\section{REFERENCIAS}

CORTEZ, P. Possíveis efeitos cognitivos e psicomotores em usuários crônicos de Cannabis. 2009.

COUTINHO, M. P. L.; ARAÚJO, L. F.; GONTIÈS, B. Uso da maconha e suas representações sociais: estudo comparativo entre universitários. Psicologia em Estudo, Maringá, v. 9, n. 3, p. 469-477, set./dez. 2004.

FERNANDES, T. F.; MONTEIRO, B. M. M.; SILVA, J. B. M.; OLIVEIRA, K. M.; VIANA, N. A. O.; GAMA, C. A. P.; GUIMARÃES, D. A. Uso de substâncias psicoativas entre universitários brasileiros: perfil epidemiológico, contextos de uso e limitações metodológicas dos estudos. Cad. Saúde Colet., 2017, Rio de Janeiro, 25 (4): 498-507.

FERRARI, C. R. Cannabis. Universidade de São Paulo. Instituto de Física de São Carlos - Psicologia da Educação - SLC 0631. Licenciatura em Ciências Exatas. 2016. $15 p$.

GARCIA, F. D. Manual de abordagem de dependências químicas. ED. Utopika, 2014, 386p.

RIBEIRO, M; MARQUES, A.C. P. R. Projeto diretrizes: Abuso e dependência Maconha. 2008.

RC: 80474

Disponível em: https://www.nucleodoconhecimento.com.br/salud/consumo-de- 
ROSA, P.; ROSA, M. G. Políticas sobre cannabis: um estudo comparativo sobre os modelos da Espanha. Geographia Opportuno Tempore Universidade Estadual de Londrina EISSN: 2358-1972, Volume 4, Número 1, 2018 Uruguai e Colorado/EUA, 2018.

SOLOWIJ, N,; PESA, N. Anormalidades cognitivas no uso da cannabis. 2010.

Enviado: Marzo de 2021.

Aprobado: Marzo de 2021.

RC: 80474

Disponível em: https://www.nucleodoconhecimento.com.br/salud/consumo-de- 